\title{
Cell palpation with an optically trapped particle
}

\author{
Tadao Sugiura $\cdot$ Hideaki Miyoshi $\cdot$ Tetsu Nishio • \\ Ayae Honda
}

Received: 1 July 2011 / Revised: 9 May 2012 / Accepted: 22 October 2012 /Published online: 9 November 2012

(C) The Author(s) 2012. This article is published with open access at Springerlink.com

\begin{abstract}
We have developed a cell palpation system to investigate cell stiffness from the reaction force generated on a particle that is fixed on a cell. In this method, a particle is used as a probe, and is manipulated towards a cell using optical tweezers. Using this method, we can obtain information of local stiffness of a cell. We investigate focal adhesion formation of a cell probed by a particle and we report different particle coating utilized for attaching certain protein in cell membrane. Also we discuss the effects of endocytosis.
\end{abstract}

Keywords Optical tweezers · Cell stiffness ·

Micromanipulation

\section{Introduction}

The cell's cytoskeleton, which is consists of proteins, provides the mechanical stiffness of the cell [1]. In general, cytoskeleton is related with cell maturation and disorder, and is easily affected by any defect in a cell. Cytological diagnosis is one of the clinical procedures that can identify certain cell conditions by studying its morphology affected by cytoskeletal changes, which may be caused by a disease. Thus, we believe that the cytoskeletal structure and also cell stiffness can become important parameters to identify different cell conditions. We developed a cell palpation system

T. Sugiura $(\bowtie) \cdot H$. Miyoshi $\cdot$ T. Nishio

Graduate School of Information Science,

Nara Institute of Science and Technology,

8916-5 Takayama-cho,

Ikoma, Nara 630-0192, Japan

e-mail: sugiura@is.naist.jp

A. Honda

Faculty of Bioscience and Applied Chemistry,

Housei University,

3-7-2 Kajino-machi,

Koganei, Tokyo 184-8584, Japan to investigate cell stiffness from reaction force generated on a particle fixed on the cell, by moving the particle using optical tweezers $[2,3]$. Optical tweezers is a technique to trap and to manipulate a micron-sized object by radiation pressure force exerted by a focused laser beam $[4,5]$. Optical tweezers has been utilized to measure weak force, such as molecular-molecular interactions [6-8], to investigate mechanism of force generation of kinesin on microtubule $[9,10]$, and study the mechanical property of DNA [11]. We use this technique to apply force on a particle attached on a cell and to measure the reaction force on the particle. The advantage of this method is that we can investigate the local mechanical property of the cell within the vicinity of the attached particle.

For the measurement of cell stiffness in local, the magnetic beads methods have been proposed and performed [12, 13]. However, there are some difficulties associated with this method; the position where the magnetic bead is to be attached cannot be controlled, and the bead is ingested into the cell while the bead is being oscillated for stiffness measurement. Also atomic force microscope (AFM) method has been performed by several researchers [14], although positioning of AFM probe and probe-to-cell attachment are not easily conducted. There are some other methods for investigating the mechanical properties of cells, such as micro fluidic cell method $[15,16]$ and a microtool method $[17,18]$. However, these methods are for cell level observation, and inappropriate for local mechanical property measurement.

In contrast optical tweezers method is suitable to investigate local mechanical property because this method enables us to place the particle onto a cell under optical microscope and specify measuring point in local. And we can measure the reaction force shortly after attachment to avoid endocytosis. The optical tweezers method has been performed also by other groups $[19,20]$. Our cell palpation system has significance on quantitative measurement capability and sensitivity to apply small force and detect weak reaction force. 
In this work we investigate the effects of cell adhesion formation and effects of different surface coatings of particles to cell palpation with optical tweezers. First we describe the principle of cell stiffness measurement with optical tweezers, and explain the actual measurement scheme in the cell palpation system. Then we report the results on the measurement of cell adhesion formation using the cell palpation system and discuss suitable timing of cell stiffness measurement.

\section{Principle of cell palpation}

In optical tweezers a small object is trapped by radiation pressure force exerted by a focused laser beam $[4,5]$. Figure 1 shows the schematic diagrams of our optical tweezers. Radiation pressure force is caused by the momentum change of light through light scattering caused by the object. Radiation force exerts the object to be pulled into a spot of the laser beam, holds the object and translates it as users wish. Objects should be dielectric particles, such as polymer particles and glass particles, for them to be manipulated by optical tweezers. Also these objects should have higher refractive indices than that of the surroundings and the range of the particle size should be from $100 \mathrm{~nm}$ to several tens micron. Since live cells consist of dielectric materials, cells can also be manipulated by optical tweezers. The exerted force is roughly proportional to the relative refractive index between objects and surroundings in cases of glass or polymer particles (refractive indices $n$ between 1.4 to 1.6) immersed in water $(n=1.33)$. Radiation force on cell membrane and cell itself are almost negligible because of very small change in refractive index.

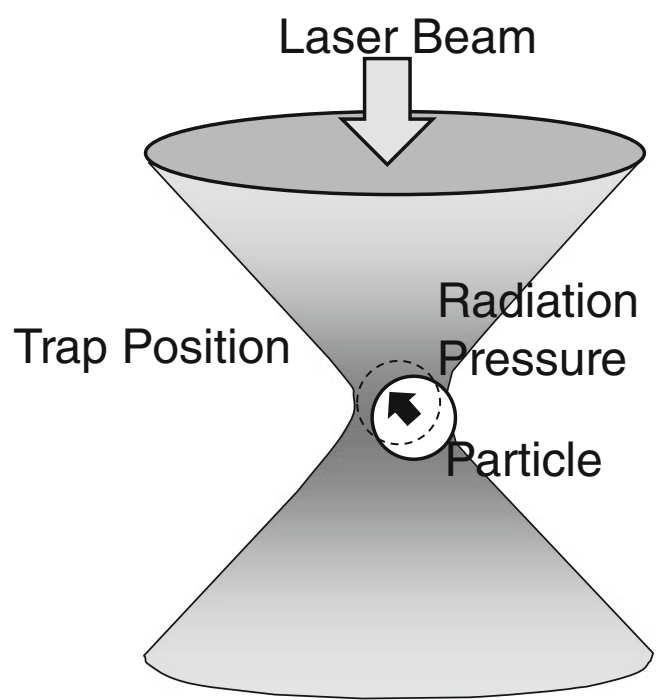

Fig. 1 Schematic diagram of optical trapping by a focused laser beam. A particle is pulled into the laser beam spot by radiation pressure force. Optical tweezers uses this phenomena to manipulate small objects
In the cell palpation system, a particle is fixed on the cell surface, and a laser beam is irradiated onto the particle, thus causing the particle to move and be used as force probe. As the particle is fixed at a point, it is observed that the particle rotates. As shown in Fig. 2, the center of the particle can be moved by shifting the laser beam spot. Exerting force on the particle is proportional to the distance $\Delta r$ between the center of the particle and the axis of the laser beam spot. When the $\Delta r$ is small enough, the force $F$ can be expressed as

$F=-k \Delta r$,

where $k$ is trapping stiffness. This relation has been utilized to measure molecular force generated by biomolecules [6-11]. Trapping stiffness can be determined before actual measurement, so the force $F$ can be estimated by measuring the distance $\Delta r$ in each moment.

Next the particle movement is conducted by applying sinusoidal displacement of laser beam spot on the particle attached on the cell surface [2]. As the particle is fixed at a location, the particle rotates and rolls up the cell membrane. This motion is considered as the particle center movement. In this condition, motion equation of the particle is described as

$$
\begin{aligned}
k\{a \sin (\omega t)-x\}= & m \frac{d^{2} x}{d t^{2}}+6 \pi \eta_{\text {med }} r \frac{d x}{d t}+r \eta_{\text {cell }} \frac{d x}{d t} \\
& +\frac{1}{2 r^{2}} Y\left(x+X_{0}\right)^{3}
\end{aligned}
$$

where $a$ and $\omega$ are oscillation amplitude and angular velocity of trapping beam, respectively. $Y$ is the cell stiffness, which represents the elastic coefficient $(\Delta F / \Delta h)$ of a cell. $h$ is the roll-up distance of cell membrane. $\eta_{\text {med }}$ and $\eta_{\text {cell }}$ are viscosity coefficients of surrounding medium and cell at the contact point of the particle. In cell palpation measurement, we move the particle in a sinusoidal manner or a rectangular manner through laser beam spot movement, and measure the position of the particle at each time $t$. Then, we can estimate the cell stiffness and viscosity coefficient of the cell from the parameters in equation (1). In actual experiments, we employ sufficiently low movement velocity as the applying force is in equilibrium with reaction force from cell as shown in equation (1). As a result, we can investigate the cell stiffness. Major contributions on the cell stiffness are from the mechanical properties of cell membrane, cell cortex, and cytoskeleton. Cell membrane and cortex consist of bilayer lipid membrane containing different kinds of proteins reinforced by membrane skeleton [1]. Cytoskeleton is regarded as a network constructed by actin and microtubule to maintain cell morphology as described by the tensegrity model [21]. These structures contribute to the mechanical property of cell. 
Fig. 2 Force generation on a particle. A particle is fixed at a point $\mathrm{P}$ and is forced to move by optical tweezers

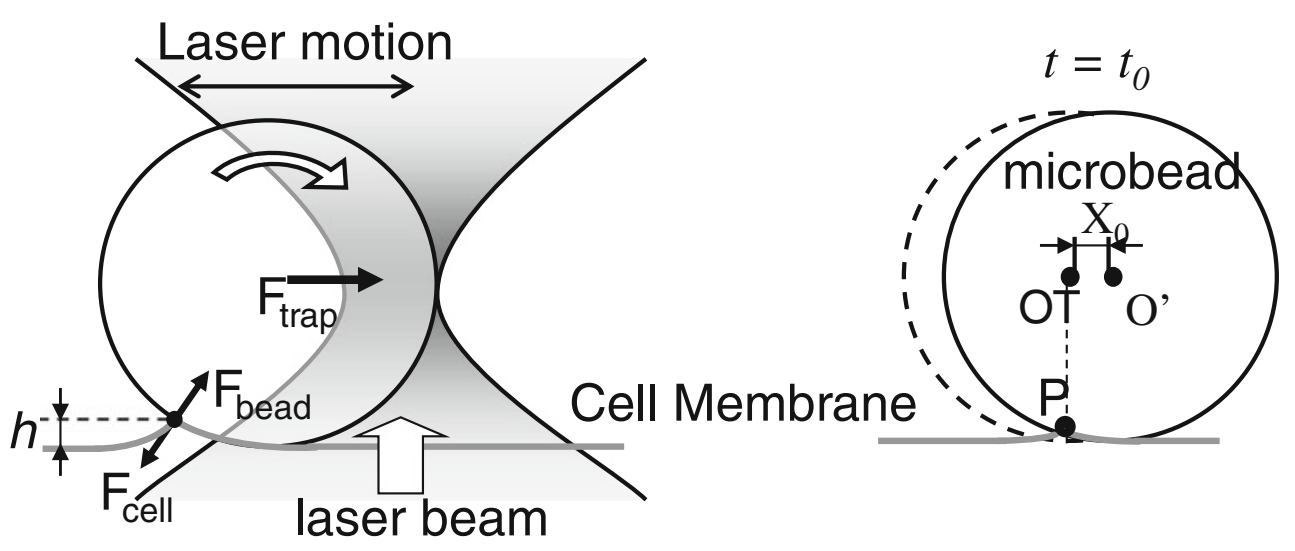

\section{Instrumentation of cell palpation system}

Figure 3 shows the schematics of cell palpation system. A laser beam from an $\mathrm{Nd}: \mathrm{YVO}_{4}$ laser (wavelength of $1064 \mathrm{~nm}, 2 \mathrm{~W}$ maximum power) is expanded with beam expander BE. The laser beam is reflected on galvanomirror $\mathrm{GM}$, is incident to microscope optics and is focused onto a sample. A particle is trapped by the laser beam, is manipulated to the desired position on the cell, and is attached onto the cell. The cell is incubated for a certain time (typically $30 \mathrm{~min}$ ), and then the cell stiffness is measured. The particle is moved by changing the angle of galvanomirror controlled by a personal computer. The resolution and accuracy of particle positioning are $10 \mathrm{~nm}$ and $4 \mathrm{~nm}$, respectively. The movement of the particle is observed through microscope optics and the images are taken by a CCD camera. The image is sent to a personal computer to undergo analysis. The particle position is estimated from the center of gravity after the binarization of the image.

We use a high numerical aperture water immersion objective (Nikon, NA $=1.20, \times 60$ ) for the optical tweezers. The image of the particle is magnified with relay lens $(\times 4)$ and imaged on a $1 / 3$ inch CCD camera. A pixel size of CCD is equivalent to $30 \mathrm{~nm}$ under the microscope. Due to the use of sub-pixel resolution capability of position estimation and reduction of unevenness of illumination, we achieved $4 \mathrm{~nm}$ of noise equivalent position fluctuation. Using the developed setup, we can measure the precise position of trapped particle.

\section{Cell measurement}

Figure 4 shows an example of position measurement. The particle that we used is a collagen coated 2 micron diameter polystyrene particle. The particle is fixed on a mouse fibroblast cell (Balb $3 \mathrm{~T} 3$ ) and is moved in a sinusoidal manner. Collagen is the main component of extra-cellular matrix and binds the integrin that exists on the cell membrane. After fixing the particle on the cell surface, the particle is anchored on the cytoskeleton, because the integrin that forms the focal adhesion is bound to the cytoskeleton. Under this condition the movement in lateral direction is restricted and

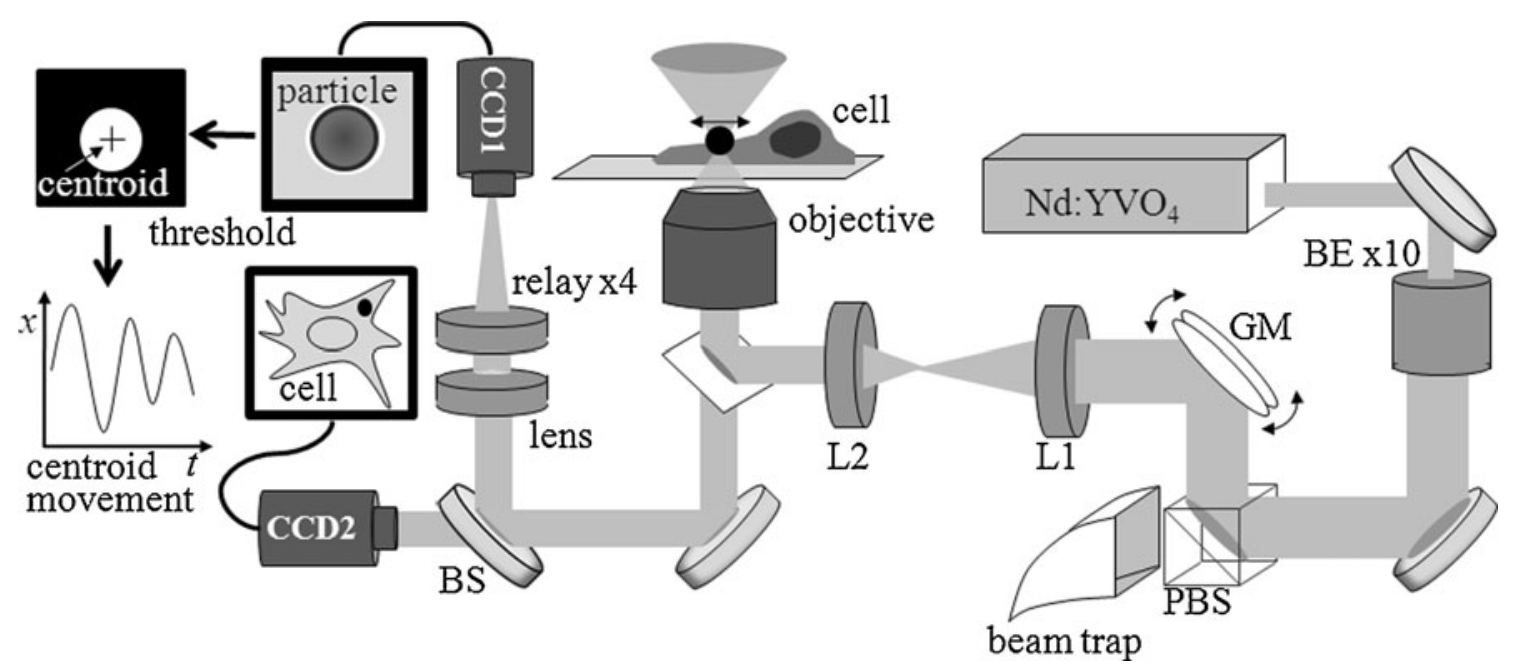

Fig. 3 Optical setup of cell palpation system. A laser beam from $\mathrm{Nd}$ : $\mathrm{YVO}_{4}$ laser (wavelength of $1064 \mathrm{~nm}$ ) is introduced into an inverted microscope setup. BE: beam expander, PBS: polarizing beam splitter,
GM: galvanomirrors, L1 and L2: lenses, BS: beam splitter. CCD1 is used for the measurement of particle position and CCD2 is for the observation of cell 
(a)

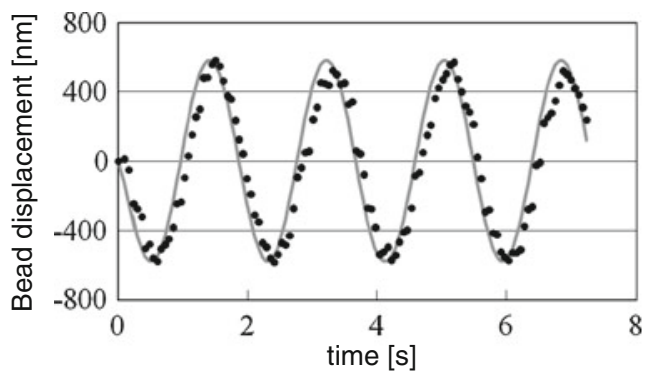

(b)

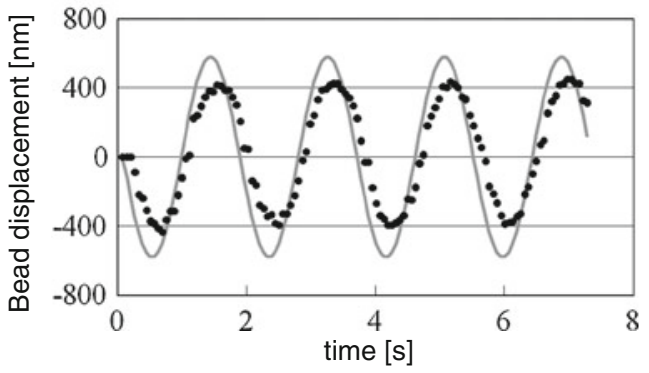

Fig. 4 Examples of particle movement. A free particle (a) and a fixed particle (b) are shown. Solid lines indicate trajectory of laser spot

the particle rolls up on the cell membrane if the particle is forced to move by optical tweezers.

Although in Fig. 4, the decrease of oscillation amplitude in four cycles of movement is unclear, the oscillation amplitude will gradually decrease as it continuously oscillates the particle. That is because an adhesion site (focal adhesion, FA) maturates thus increasing the cell stiffness, and the number of collagen molecules the bind to integrin increases as particle rotate on cell surface.

By considering the attachment of particle on the cell surface, there are two possible measurement modes for cell palpation. One mode is to put and oscillate a particle on a cell surface and measure the particle movement continuously (mode 1). The other mode is to put the particle and wait a certain time, then move it back and forth once and measure the particle position (mode 2). These modes will give different types of cell property on mechanics. In mode 1, new bond formation and bond rapture always occur during oscillation, so we can investigate one bond formation and rapture as stepwise change in oscillation amplitude. From this data, we can estimate one bond contribution on total adhesion phenomena. Also, we induce mechanical stimuli on the cell through the oscillating movement of particle. From this, we can identify the cellular mechanical response as a result of the mechanical stimuli $[22,23]$. In mode 2, we can investigate natural mechanical properties of cells because no reactive response is induced on the cell. We need to select suitable measurement mode in actual situation. In this work, we aim to investigate cell adhesion formation and effect of surface coating of particles to cell palpation. For

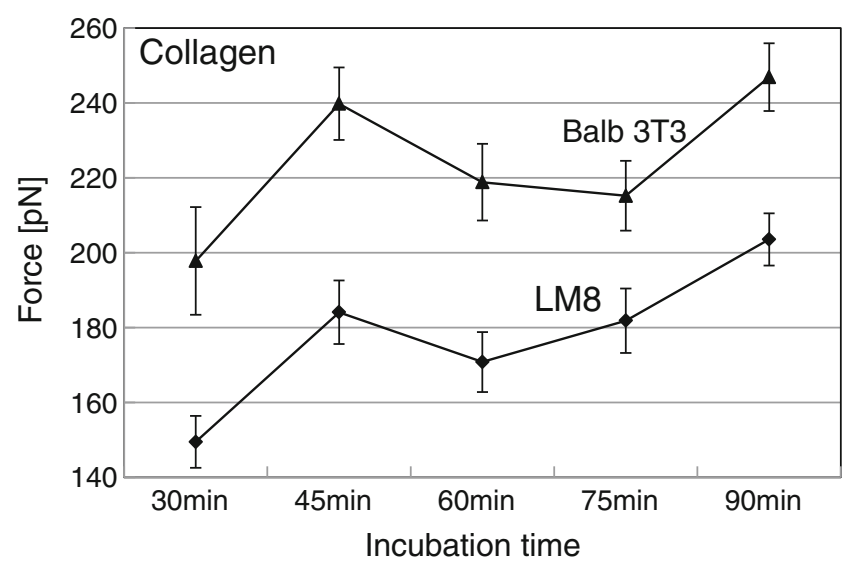

Fig. 5 Time courses of focal adhesion maturation observed by cell palpation system. Two types of cells show the similar time course

this reason, we used mode 2 measurement in following experiments. Also once a particle attached on cell surface this particle hardly removed from the cell, because of bond formation between surfaces of cell and particle. Thus we usually do not reuse a particle after one measurement, but utilize a new particle floating around cells as a probe for next measurement every time.

\section{Cell adhesion formation measurement}

A cell forms complex adhesion structure through contact with other cells or substrates. The adhesion structure is called focal adhesion (FA). FA is important to stabilize a cell at a certain location and it plays an important role on cell migration and cancer metastasis. We employed the mode 2 scheme to investigate the time course of focal adhesion formation by changing the elapsed time $t$ to a single back-and-forth movement after putting a particle on

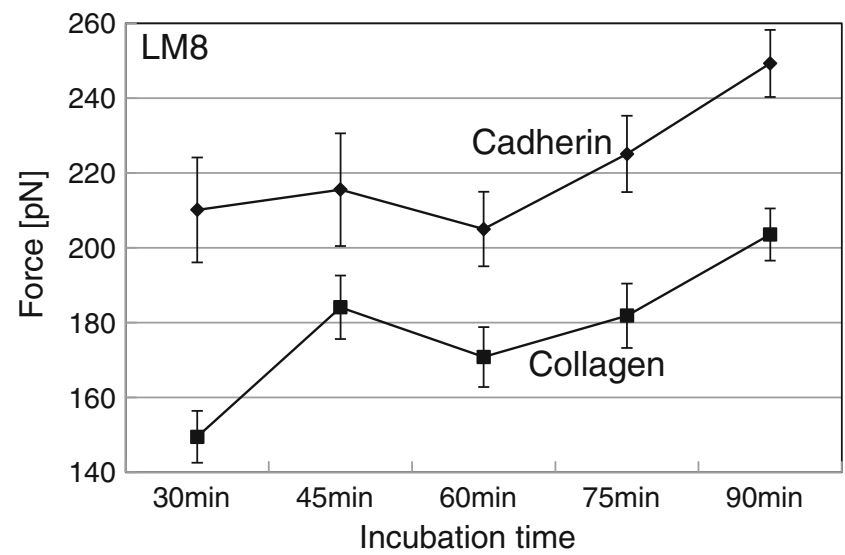

Fig. 6 Dependency on binding site in cell palpation measurement. Cadherin binding particle represents immediate increase of cell stiffness, although collagen-coated particle shows gradual maturation of focal adhesion (same in Fig. 5) 
the cell surface. For each elapsed time, we measured 20 cells. Cells are mouse fibroblast cell (Balb 3 T3) and mouse osteosarcoma cell (LM8). For the particles, we have employed 2-micron diameter polystyrene beads coated with collagen. The particles were moved in $400 \mathrm{~nm}$ from a contact point by use of optical tweezers, which had a spring constant $k$ of $4 \mathrm{mN} / \mathrm{m}$, and measured the magnitude of reaction force from the cell. In this measurement resolution and accuracy of reaction force measurement are estimated as 2 $\mathrm{pN}$ and $16 \mathrm{pN}$, respectively.

To ensure strong binding between collagen and particle surface, we used carboxyl polystyrene particle (Spherotech Inc., CP-20-10) and formed covalent bond between first amino group of collagen and carboxyl group by use of cross-linker. Cells are dispersed on glass bottom dish 24 to 36 hours before experiment. The glass bottom dish is coated with collagen to bind tightly. Finally the cells are incubated after putting the particles on the cell surface in $37^{\circ} \mathrm{C}, 5 \%$ $\mathrm{CO}_{2}$ atmosphere.

Figure 5 shows the results as mean values with standard deviations as error bars. Cell stiffness is expressed as a value of reaction force when a 2-micron diameter particle is moved. In the process of focal adhesion formation, during the first $45 \mathrm{~min}$, cell stiffness increases gradually; at around 60 and $75 \mathrm{~min}$, the stiffness decreases, and then increases again. This result is interpreted as the corresponding time course of initial adhesion formation investigated with molecular biology method. Although the mechanism of the difference in the values of fibroblast cells and osteosarcoma cells is still unclear, we speculate that the difference comes from the structural discrepancy of the cell membranes.

The increase in cell stiffness after 75 min may be due to the ingestion of the particle through endocytosis. According to this, we should better choose $60 \mathrm{~min}$ after particle contact to the cell surface can avoid initial adhesion formation and endocytosis. In addition, since this condition is expected to depend on cell types, we may choose the suitable condition for the duration of adhesion maturation.

\section{Effect of surface coating on particle}

Although in previous experiment we have used a collagen coated particle for cell palpation, we expect different coating materials that bind to different target sites can provide additional information on cell stiffness. Therefore, we have also done experiments using particles with different coatings. We have chosen cadherin as the target site, which exists widely on cell membrane [1]. Cadherin is not a force sensitive protein, as opposed to integrin, which is a force sensing protein and may cause reaction against applied force.

To bind a particle to cadherin we used anti-cadherin antibody fixed on particles. Similar to previous experiments, we have used cross-linkers to form covalent bond between antibody and particle surface. The particles used are 2micron diameter polystyrene and cells are mouse osteosarcoma cell LM8.

Figure 6 shows the results as mean values with standard deviations as error bars. For comparison, the results of the collagen coated beads are also shown. Stiffness value of cadherin case is slightly larger, because we suppose that the number of cadherin site is larger than that of the integrin. Also the value within $30 \mathrm{~min}$ is already almost plateau. This is because anti-cadherin antibody binds to cadherin protein immediately after touching, and the contact structure is not enhanced by applying external forces. After $75 \mathrm{~min}$, the stiffness value increases, because of endocytosis. We conclude that anti-cadherin antibody coating particle is more suitable for cell palpation, because there is no induced growth effect on adhesion in case of cadherin.

\section{Conclusion}

We developed the cell palpation system, which is applicable to adhesive cells. In current system, we can measure cell stiffness and investigate the maturation of cell adhesion processes. Advantages of using optical tweezers are the ease of probe particle manipulation, flexibility in specifying which cell location to place the particle, and immediate measurement of force reaction.

We believe cell stiffness is an important parameter on monitoring cell activity and malignancy of cancer cells or other diseases related to cells. We expect the cell palpation system to be utilized as a useful tool for future diagnostics in the medical field.

Open Access This article is distributed under the terms of the Creative Commons Attribution License which permits any use, distribution, and reproduction in any medium, provided the original author (s) and the source are credited.

\section{References}

1. Alberts B, Johnson A, Lewis J, Raff M (2002) Molecular biology of the cell, 4th edn. Garland Science, New York

2. Miyoshi H, Sugiura T, Minato K (2009) Cell palpation system based on a force measurement by optical tweezers for investigation of local mechanical properties of a cell membrane. Jap J Appl Phys 48:120223-1-3

3. Sugiura T, Nakao M, Sato T, Minato K (2008) Nanomanipulation and nanotechnology for future diagnostics. In: eHealth: Combining health telecatics, telemedicine, biomedical engineering and bioinformatics to the edge. IOS Press, Amsterdam, pp 135-142 
4. Ashkin A (1986) Obserbation of a single-beam gradient force optical trap for dielectric particles. Opt Lett 11:288-290

5. Ashkin A (1987) Optical trapping and manipulation of single cells using infrared-laser beams. Nature 330:769-771

6. Ota T, Sugiura T, Kawata S (2005) Rupture-force measurement of biotin-streptavidin bonds using optical trapping. Appl Phys Lett 87:043901-1-3

7. Finer JT (1994) Single myosin molecule mechanics: piconewton forces and nanometer steps. Nature 368:113-119

8. Yin H (1995) Transcripiton against an applied force. Science 270:1653-1657

9. Guydosh NR, Block SM (2009) Direct observation of the binding state of the kinesin head to the microtubule. Nature 461:125-128

10. Lang MJ, Fordyce PM, Engh AM, Neuman KC, Block SM (2004) Simultaneous coincident optical trapping and single-molecule fluorescence. Nat Methods 1:133-139

11. Smith SB, Cui Y, Bustamante C (1996) Overstretching B-dna: the elastic response of individual double-stranded and single-stranded dna molecules. Science 271:795-799

12. Fabry B, Maksym GN, Butler JP, Glogauer M, Navajas D, Fredberg JJ (2001) Scaling the microrheology of living cells. Phys Rev Lett 87:148102-1-4

13. Kollmannsberger P, Fabry B (2007) High-force magnetic tweezers with force feedback for biological applications. Rev Sci Instr 78:114301-1-6

14. Lam WA, Rosenbluth MJ, Fletcher DA (2007) Chemotherapy exposure increases leukemia cell stiffness. Blood 109:35053508

15. Ahmad MR, Nakajima M, Kojima S, Homma M, Fukuda T (2008) In situ single cell mechanics characterization of yeast cells using nanoneedles inside environmental SEM. IEEE Trans Nanotech 7:607-616

16. Uchida R, Tanaka N, Higashimori M, Tadakuma K, Kaneko M, Kondo M, Yamato M (2010) Cell Sheet Stiffness Sensing without taking out from culture liquid. Annual International Conference of the IEEE Engineering in Medicine and Biology Society (EMBC) $827-830$

17. Hirose Y, Tadakuma K, Higashimori M, Arai T, Kaneko M, Iitsuka R, Yamanishi Y, Arai F (2010) A new stiffness evaluation toward high speed cell sorter. IEEE International Conference on Robotics and Automation (ICRA), 4113-4118

18. Kawahara T, Hagiwara M, Yamanishi Y, Arai F (2010) Control and sensing platform of magnetically driven microtool for on-chip single cell evaluation. International Symposium on Micro-Nano Mechatronics and Human Science (MHS) 322-327

19. lkuta K, Sato F, Kadoguchi K, Itoh S (2008) Optical driven masterslave controllable nano-manipulator with real-time force sensing. IEEE International Conference on Micro Electro Mechanical Systems (MEMS) 539-542

20. Youhua T, Dong S, Shuk HC, Li RA (2011) Robotic cell manipulation with optical tweezers for biomechanical characterization. IEEE International Conference on Robotics and Automation (ICRA) 4104-4109

21. Ingber DE (2003) Tensegrity I. Cell structure and hierarchical systems biology. J Cell Sci 116:1157-1173

22. Vogel V, Sheetz MP (2009) Cell fate regulation by coupling mechanical cycles to biochemical signaling pathways. Curr Opin Cell Biol 21:38-46

23. Khan S, Sheetz MP (1997) Force effects on biochemical kinetics. Annu Rev Biochem 66:785-805 